\title{
THREE-SHEETED ALGEBROID SURFACES WHOSE PICARD CONSTANTS ARE FIVE
}

\author{
Dedicated to Professor Nobuyuki Suita on his 60th birthday \\ By Mitsuru Ozawa AND KazUnari SAwada
}

\section{§1. Introduction}

The notion of Picard constant of a Riemann surface $R$ was introduced in [3]. Let $\mathscr{M}(R)$ be the family of non-constant meromorphic functions on $R$. Let $P(f)$ be the number of values which are not taken by $f$ in $\mathscr{M}(R)$. Now put

$$
P(R)=\sup _{f \in \mathcal{M}(R)} P(f) .
$$

This $P(R)$ is called the Picard constant of $R$. If $R$ is open, then $P(R) \geqq 2$. Further if $R$ is an $n$-sheeted algebroid surface, which is the proper existence domain of an $n$-valued algebroid function, then $P(R) \leqq 2 n$ by Selberg's theory of algebroid functions [7].

We now list up two results for the case of three-sheeted algebroid surfaces. The first one is the following: Let $R$ be a regularly branched three-sheeted algebroid surface, that is, $R$ is defined by $y^{3}=g(x)$, where $g(x)$ is an entire function with infinitely many simple or double zeros. Then $P(R)=6$, if and only if $g(x)=\left(e^{H}-\alpha\right)\left(e^{H}-\beta\right)^{2}, \alpha \beta(\alpha-\beta) \neq 0$, where $H$ is a non-constant entire function with $H(0)=0$ and $\alpha, \beta$ are constants. Further there is no regularly branched three-sheeted surface $R$ with $P(R)=5$ [1].

The second one is the following: Let $R$ be a general three-sheeted algebroid surface. Then there are two kinds of surfaces $R$ with $P(R)=6$. One is defined by

$$
y^{3}-\left(x_{0} e^{H}+x_{1}\right) y^{2}+\left(a_{1} x_{0} e^{H}+x_{2}\right) y-x_{8}=0,
$$

where $x_{0}$ is a non-zero constant, $x_{1}=a_{2}+a_{3}+a_{4}, x_{2}=a_{2} a_{3}+a_{3} a_{4}+a_{2} a_{4}$ and $x_{3}=$ $a_{2} a_{3} a_{4}$ with non-zero different complex numbers $a_{1}, a_{2}, a_{3}, a_{4}$ and $H$ is a nonconstant entire function with $H(0)=0$. The other is defined by

$$
y^{3}-\left(x_{0} e^{H}+x_{1}\right) y^{2}+\left\{\left(a_{1}+a_{2}\right) x_{0} e^{H}+x_{2}\right\} y-a_{1} a_{2} x_{0} e^{H}=0,
$$

where $x_{0}$ is a non-zero constant, $x_{1}=a_{3}+a_{4}, x_{2}=a_{3} a_{4}$ with non-zero different complex numbers $a_{1}, a_{2}, a_{3}, a_{4}$ and $H$ is a non-constant entire function with $H(0)=0[5]$.

Received July 15, 1992; revised August 23, 1993. 
In general it is very difficult to decide the exact value of $P(R)$ of any given surface $R$. Our problem is the following one: Is there any method to prove $P(R)=5$ for three-sheeted algebroid surfaces? In the first place we shall determine several three-sheeted algebroid surfaces $R$ :

$$
y^{3}-S_{1} y^{2}+S_{2} y-S_{3}=0
$$

with $P(y)=5$. Then we shall give a method to prove really $P(R)=5$.

\section{§2. Surfaces with $P(y)=5$}

Let us put

$$
F(z, y) \equiv y^{3}-S_{1} y^{2}+S_{2} y-S_{3} .
$$

By Rémoundos' theorem [6] we may consider firstly

$$
\left(\begin{array}{l}
F(z, 0) \\
F\left(z, a_{2}\right) \\
F\left(z, a_{3}\right) \\
F\left(z, a_{4}\right)
\end{array}\right)=\left(\begin{array}{c}
c_{1} \\
\beta_{1} e^{H_{1}} \\
\beta_{2} e^{H_{2}} \\
\beta_{3} e^{H_{3}}
\end{array}\right) \text { or }\left(\begin{array}{c}
\beta_{1} e^{H_{1}} \\
c_{1} \\
\beta_{2} e^{H_{2}} \\
\beta_{3} e^{H_{3}}
\end{array}\right) \text {, }
$$

where $c_{1}, \beta_{1}, \beta_{2}, \beta_{3}$ are non-zero constants and $H_{1}, H_{2}, H_{3}$ are non-constant entire functions satisfying $H_{1}(0)=H_{2}(0)=H_{3}(0)=0$. The first one is the same as the following simultaneous equation:

$$
\left\{\begin{aligned}
-S_{3} & =c_{1}, \\
a_{2}{ }^{3}-S_{1} a_{2}{ }^{2}+S_{2} a_{2}-S_{3} & =\beta_{1} e^{H_{1}}, \\
a_{3}{ }^{3}-S_{1} a_{3}{ }^{2}+S_{2} a_{3}-S_{3} & =\beta_{2} e^{H_{2}}, \\
a_{4}{ }^{3}-S_{1} a_{4}{ }^{2}+S_{2} a_{4}-S_{3} & =\beta_{3} e^{H_{3}} .
\end{aligned}\right.
$$

Then by Borel's unicity theorem [2]

$$
c_{1}=-a_{2} a_{3} a_{4}, \quad H_{1}=H_{2}=H_{3} \equiv H
$$

and

$$
a_{2} a_{4}\left(a_{4}-a_{2}\right) \beta_{2}-a_{2} a_{3}\left(a_{3}-a_{2}\right) \beta_{3}+a_{3} a_{4}\left(a_{3}-a_{4}\right) \beta_{1}=0 \text {. }
$$

Further

$$
\left\{\begin{array}{l}
S_{1}=\frac{a_{4} \beta_{2}-a_{3} \beta_{3}}{a_{3} a_{4}\left(a_{4}-a_{3}\right)} e^{H}+a_{2}+a_{3}+a_{4}, \\
S_{2}=\frac{a_{4}{ }^{2} \beta_{2}-a_{3}{ }^{2} \beta_{3}}{a_{3} a_{4}\left(a_{4}-a_{3}\right)} e^{H}+a_{2} a_{3}+a_{3} a_{4}+a_{2} a_{4}, \\
S_{3}=-c_{1}=a_{2} a_{3} a_{4} .
\end{array}\right.
$$


Let us compute $F(z, A)$. Then

$$
\begin{aligned}
F(z, A)=A^{3}-A^{2} S_{1}+A S_{2}-S_{3}= & \frac{A\left\{\left(-A a_{4}+a_{4}{ }^{2}\right) \beta_{2}-\left(a_{3}{ }^{2}-A a_{3}\right) \beta_{3}\right\}}{a_{3} a_{4}\left(a_{4}-a_{3}\right)} e^{H} \\
& +\left(A-a_{2}\right)\left(A-a_{3}\right)\left(A-a_{4}\right) .
\end{aligned}
$$

Suppose that $F(z, A)$ does not reduce to a non-zero constant for any non-zero constant $A$. Then there is no non-zero constant $A$ for which $A\left(a_{3} \beta_{3}-a_{4} \beta_{2}\right)=$ $a_{3}{ }^{2} \beta_{3}-a_{4}{ }^{2} \beta_{2}$. Hence we have either $a_{3} \beta_{3}=a_{4} \beta_{2}$ or $a_{3}{ }^{2} \beta_{3}=a_{4}{ }^{2} \beta_{2}$. In the former case

$$
\text { (A) } \frac{\beta_{3}}{a_{4}}=\frac{\beta_{2}}{a_{3}}=\frac{\beta_{1}}{a_{2}}
$$

and in the latter case

$$
\text { (B) } \frac{\beta_{3}}{a_{4}{ }^{2}}=\frac{\beta_{2}}{a_{3}{ }^{2}}=\frac{\beta_{1}}{a_{2}{ }^{2}} \text {. }
$$

Case (A). Then

$$
\left\{\begin{array}{l}
S_{1}=a_{2}+a_{3}+a_{4} \equiv y_{1}, \\
S_{2}=y_{0} e^{H}+a_{2} a_{3}+a_{3} a_{4}+a_{2} a_{4} \equiv y_{0} e^{H}+y_{2}, \\
S_{3}=a_{2} a_{3} a_{4} \equiv y_{3}
\end{array}\right.
$$

with $y_{0}=\beta_{2} / a_{3}$. Let us consider the discriminant of $R: y^{3}-S_{1} y^{2}+S_{2} y-S_{3}=0$. Let us denote it by $\Delta$, then

$$
\begin{aligned}
\Delta & =4 S_{1}{ }^{3} S_{3}-S_{1}{ }^{2} S_{2}{ }^{2}-18 S_{1} S_{2} S_{3}+4 S_{2}{ }^{3}+27 S_{3}{ }^{2} \\
& =4 y_{0}{ }^{3} e^{3 H}+\zeta_{2} y_{0}{ }^{2} e^{2 H}+\zeta_{1} y_{0} e^{H}+\zeta_{0},
\end{aligned}
$$

where $\zeta_{2}=12 y_{2}-y_{1}{ }^{2}, \zeta_{1}=12 y_{2}{ }^{2}-18 y_{1} y_{3}-2 y_{1}^{2} y_{2}$ and $\zeta_{0}=4 y_{1}^{3} y_{3}-y_{1}{ }^{2} y_{2}{ }^{2}-18 y_{1} y_{2} y_{3}$ $+4 y_{2}{ }^{3}+27 y_{3}{ }^{2}$, which is equal to $-\left(a_{2}-a_{3}\right)^{2}\left(a_{3}-a_{4}\right)^{2}\left(a_{2}-a_{4}\right)^{2} \neq 0$. We denote this surface by $R_{A}$.

Case (B). Then with the same notations $y_{1}, y_{2}, y_{3}$ as in (A)

$$
\left\{\begin{array}{l}
S_{1}=y_{0} e^{H}+y_{1}, \quad y_{0}=-\beta_{2} / a_{3}{ }^{2}, \\
S_{2}=y_{2} \\
S_{3}=y_{3} .
\end{array}\right.
$$

In this case the discriminant $\Delta$ of $R$ is

$$
\Delta=4 y_{0}{ }^{3} e^{3 H} y_{3}+\zeta_{2} y_{0}{ }^{2} e^{2 H}+\zeta_{1} y_{0} e^{H}+\zeta_{0},
$$

where $\zeta_{2}=12 y_{1} y_{3}-y_{2}{ }^{2}, \quad \zeta_{1}=12 y_{1}{ }^{2} y_{3}-2 y_{1} y_{2}{ }^{2}-18 y_{2} y_{3}$ and $\zeta_{0}=4 y_{1}{ }^{3} y_{3}-y_{1}{ }^{2} y_{2}{ }^{2}-$ $18 y_{1} y_{2} y_{3}+4 y_{2}^{3}+27 y_{3}^{2}$, which is equal to $-\left(a_{2}-a_{3}\right)^{2}\left(a_{3}-a_{4}\right)^{2}\left(a_{2}-a_{4}\right)^{2} \neq 0$. We 
denote this surface by $R_{B}$.

The second one is the same as the following simultaneous equation:

$$
\left\{\begin{aligned}
-S_{3} & =\beta_{1} e^{H_{1}}, \\
a_{2}{ }^{3}-S_{1} a_{2}{ }^{2}+S_{2} a_{2}-S_{3} & =c_{1}, \\
a_{3}{ }^{3}-S_{1} a_{3}{ }^{2}+S_{2} a_{3}-S_{3} & =\beta_{2} e^{H_{2}}, \\
a_{4}{ }^{3}-S_{1} a_{4}{ }^{2}+S_{2} a_{4}-S_{3} & =\beta_{3} e^{H_{3}} .
\end{aligned}\right.
$$

By Borel's unicity theorem

and

$$
\begin{aligned}
& c_{1}=a_{2}\left(a_{2}-a_{3}\right)\left(a_{2}-a_{4}\right), \\
& H_{1}=H_{2}=H_{3} \equiv H
\end{aligned}
$$

$$
a_{2} a_{4}\left(a_{4}-a_{2}\right) \beta_{2}+\left(a_{2}-a_{3}\right)\left(a_{4}-a_{2}\right)\left(a_{3}-a_{4}\right) \beta_{1}+a_{2} a_{3}\left(a_{2}-a_{3}\right) \beta_{3}=0 .
$$

Then we have

$$
\left\{\begin{array}{l}
S_{1}=\frac{e^{H}}{a_{2} a_{3}\left(a_{2}-a_{3}\right)}\left(a_{2} \beta_{2}-\left(a_{2}-a_{3}\right) \beta_{1}\right)+a_{3}+a_{4}, \\
S_{2}=\frac{e^{H}}{a_{2} a_{3}\left(a_{2}-a_{3}\right)}\left(a_{2}{ }^{2} \beta_{2}-\left(a_{2}{ }^{2}-a_{3}{ }^{2}\right) \beta_{1}\right)+a_{3} a_{4}, \\
S_{3}=-\beta_{1} e^{H} .
\end{array}\right.
$$

Now we pose the following condition: There is no non-zero constant $A$, being different from $a_{2}$, such that $F(z, A)$ reduces to a non-zero constant. In this case

$$
\begin{aligned}
F(z, A)= & \frac{e^{H}}{a_{2} a_{3}\left(a_{2}-a_{3}\right)}\left\{-A^{2}\left(a_{2} \beta_{2}-\left(a_{2}-a_{3}\right) \beta_{1}\right)+A\left(a_{2}{ }^{2} \beta_{2}-\left(a_{2}{ }^{2}-a_{3}{ }^{2}\right) \beta_{1}\right)\right. \\
& \left.+a_{2} a_{3}\left(a_{2}-a_{3}\right) \beta_{1}\right\}+A\left(A-a_{3}\right)\left(A-a_{4}\right)
\end{aligned}
$$

dose not reduce to a non-zero constant excepting $A=a_{2}$.

Case (C). $-A^{2}\left(a_{2} \beta_{2}-\left(a_{2}-a_{3}\right) \beta_{1}\right)+A\left(a_{2}^{2} \beta_{2}-\left(a_{2}{ }^{2}-a_{3}{ }^{2}\right) \beta_{1}\right)+a_{2} a_{3}\left(a_{2}-a_{3}\right) \beta_{1}=$ $\alpha\left(A-a_{2}\right)^{2}$ with some constant $\alpha \div 0$. Then

$$
\left\{a_{2}{ }^{2} \beta_{2}-\left(a_{2}{ }^{2}-a_{3}{ }^{2}\right) \beta_{1}\right\}^{2}=-4\left\{a_{2} \beta_{2}-\left(a_{2}-a_{3}\right) \beta_{1}\right\} a_{2} a_{3}\left(a_{2}-a_{3}\right) \beta_{1},
$$

which implies

Then

$$
a_{2}^{2} \beta_{2}=\left(a_{2}-a_{3}\right)^{2} \beta_{1}
$$

$$
F(z, A)=\frac{\beta_{1} e^{H}}{a_{2}{ }^{2}}\left(A-a_{2}\right)^{2}+A\left(A-a_{3}\right)\left(A-a_{4}\right) .
$$

In this case we have 


$$
\left\{\begin{array}{l}
S_{1}=y_{0} e^{H}+y_{1}, \quad y_{0}=-\beta_{1} / a_{2}{ }^{2}, \\
S_{2}=2 a_{2} y_{0} e^{H}+y_{2}, \quad y_{1}=a_{3}+a_{4}, \quad y_{2}=a_{3} a_{4}, \\
S_{3}=a_{2}{ }^{2} y_{0} e^{H} .
\end{array}\right.
$$

Then the discriminant $\Delta$ of $y^{3}-S_{1} y^{2}+S_{2} y-S_{3}=0$ is

$$
\Delta=\xi_{3} y_{0}{ }^{3} e^{3 H}+\xi_{2} y_{0}{ }^{2} e^{2 H}+\xi_{1} y_{0} e^{H}+\xi_{0},
$$

where

$$
\begin{aligned}
& \xi_{3}=4\left(a_{2}{ }^{2} y_{1}-a_{2}{ }^{3}-a_{2} y_{2}\right)=-4 a_{2}\left(a_{2}-a_{3}\right)\left(a_{2}-a_{4}\right) \neq 0, \\
& \xi_{2}=8 a_{2}{ }^{2} y_{1}{ }^{2}-36 a_{2}{ }^{3} y_{1}+27 a_{2}{ }^{4}-8 a_{2} y_{1} y_{2}+30 a_{2}{ }^{2} y_{2}-y_{2}{ }^{2}, \\
& \xi_{1}=4 a_{2}{ }^{2} y_{1}{ }^{3}-4 a_{2} y_{1}{ }^{2} y_{2}-18 a_{2}{ }^{2} y_{1} y_{2}-2 y_{1} y_{2}{ }^{2}+24 a_{2} y_{2}{ }^{2}, \\
& \xi_{0}=-y_{2}{ }^{2}\left(y_{1}{ }^{2}-2 y_{2}\right)=-a_{3}{ }^{2} a_{4}{ }^{2}\left(a_{3}-a_{4}\right)^{2} \neq 0 .
\end{aligned}
$$

We denote this surface by $R_{c}$.

Case (D). $\quad-A^{2}\left(a_{2} \beta_{2}-\left(a_{2}-a_{3}\right) \beta_{1}\right)+A\left(a_{2}{ }^{2} \beta_{2}-\left(a_{2}{ }^{2}-a_{3}{ }^{2}\right) \beta_{1}\right)+a_{2} a_{3}\left(a_{2}-a_{3}\right) \beta_{1}=$ $\alpha\left(A-a_{2}\right)$ with some non-zero constant $\alpha$ being independent of $A$. Then $a_{2} \beta_{2}=$ $\left(a_{2}-a_{3}\right) \beta_{1}$ firstly and hence the above expression is equal to $-a_{3}\left(a_{2}-a_{3}\right) \beta_{1}\left(A-a_{2}\right)$. Then we have

$$
F(z, A)=\frac{a_{2}-A}{a_{2}} \beta_{1} e^{H}+A\left(A-a_{3}\right)\left(A-a_{4}\right) .
$$

In this case we have

$$
\left\{\begin{array}{l}
S_{1}=y_{1}, \quad y_{0}=-\beta_{1} / a_{2}, \\
S_{2}=y_{0} e^{H}+y_{2}, \quad y_{1}=a_{3}+a_{4}, \quad y_{2}=a_{3} a_{4}, \\
S_{3}=a_{2} y_{0} e^{H} .
\end{array}\right.
$$

Then the discriminant $\Delta$ of $y^{3}-S_{1} y^{2}+S_{2} y-S_{3}=0$ is

$$
\Delta=4 y_{0}{ }^{3} e^{3 H}+\xi_{2} y_{0}{ }^{2} e^{2 H}+\xi_{1} y_{0} e^{H}+\xi_{0},
$$

where

$$
\begin{aligned}
& \xi_{2}=12 y_{2}+27 a_{2}{ }^{2}-18 a_{2} y_{1}-y_{1}{ }^{2}, \\
& \xi_{1}=12 y_{2}{ }^{2}-18 a_{2} y_{1} y_{2}-6 y_{1}{ }^{2} y_{2}+4 a_{2} y_{1}{ }^{3}, \\
& \xi_{0}=y_{2}{ }^{2}\left(4 y_{2}-y_{1}{ }^{2}\right)=-a_{3}{ }^{2} a_{4}{ }^{2}\left(a_{3}-a_{4}\right)^{2} \neq 0 .
\end{aligned}
$$

We denote this surface by $R_{D}$.

We now consider 


$$
\left(\begin{array}{l}
F(z, 0) \\
F\left(z, a_{1}\right) \\
F\left(z, a_{2}\right) \\
F\left(z, a_{3}\right)
\end{array}\right)=\left(\begin{array}{c}
c_{1} \\
c_{2} \\
\beta_{1} e^{H_{1}} \\
\beta_{2} e^{H_{2}}
\end{array}\right) \text { or }\left(\begin{array}{c}
\beta_{1} e^{H_{1}} \\
c_{1} \\
c_{2} \\
\beta_{2} e^{H_{2}}
\end{array}\right)
$$

The first one is the following simultaneous equation:

$$
\left\{\begin{aligned}
-S_{3} & =c_{1}, \\
a_{1}{ }^{3}-S_{1} a_{1}{ }^{2}+S_{2} a_{1}-S_{3} & =c_{2}, \\
a_{2}{ }^{3}-S_{1} a_{2}{ }^{2}+S_{2} a_{2}-S_{3} & =\beta_{1} e^{H_{1}}, \\
a_{3}{ }^{3}-S_{1} a_{3}{ }^{2}+S_{2} a_{3}-S_{3} & =\beta_{2} e^{H_{2}} .
\end{aligned}\right.
$$

By Borel's unicity theorem $H_{1}=H_{2} \equiv H, a_{3}\left(a_{3}-a_{1}\right) \beta_{1}=a_{2}\left(a_{2}-a_{1}\right) \beta_{2}$ and

Then

$$
c_{1}\left(a_{3}-a_{1}\right)\left(a_{2}-a_{1}\right)-c_{2} a_{2} a_{3}+a_{1} a_{2} a_{3}\left(a_{3}-a_{1}\right)\left(a_{2}-a_{1}\right)=0 .
$$

$$
\left\{\begin{array}{l}
S_{1}=-\frac{c_{1}}{a_{2} a_{3}}+a_{2}+a_{3}-\frac{\beta_{1} e^{H}}{a_{2}\left(a_{2}-a_{1}\right)}, \\
S_{1}=-\frac{\left(a_{2}+a_{3}\right) c_{1}}{a_{2} a_{3}}+a_{2} a_{3}-\frac{a_{1} \beta_{1} e^{H}}{a_{2}\left(a_{2}-a_{1}\right)}, \\
S_{3}=-c_{1} .
\end{array}\right.
$$

Now we pose the following condition: There is no non-zero constant $B$, being different from $a_{2}$ and $a_{3}$, such that $F(z, B)$ reduces to the form $\alpha e^{X}$, where $\alpha \neq 0$ and $X$ : non-constant entire function.

$$
F(z, B)=\frac{B\left(B-a_{1}\right)}{a_{2}\left(a_{2}-a_{1}\right)} \beta_{1} e^{H}+\frac{\left(B-a_{2}\right)\left(B-a_{3}\right)}{a_{2} a_{3}}\left(c_{1}+B a_{2} a_{3}\right) .
$$

Case (E). $\quad c_{1}+B a_{2} a_{3}=a_{2} a_{3}\left(B-a_{2}\right)$. Then $c_{1}=-a_{2}{ }^{2} a_{3}$ and $c_{2}=-\left(a_{3}-a_{1}\right)\left(a_{2}\right.$ $\left.-a_{1}\right)^{2}$. Further

$$
\left\{\begin{array}{l}
S_{1}=2 a_{2}+a_{3}+y_{0} e^{H}, \quad y_{0}=-\frac{\beta_{1}}{a_{2}\left(a_{2}-a_{1}\right)}, \\
S_{2}=a_{2}{ }^{2}+2 a_{2} a_{3}+a_{1} y_{0} e^{H}, \\
S_{3}=-c_{1}=a_{2}^{2} a_{3} .
\end{array}\right.
$$

In this case the discriminant $\Delta$ of $y^{3}-S_{1} y^{2}+S_{2} y-S_{3}=0$ is

$$
\Delta=y_{0} e^{H}\left(-a_{1}^{2} y_{0}^{3} e^{3 H}+A_{2} y_{0}{ }^{2} e^{2 H}+A_{1} y_{0} e^{H}+A_{0}\right),
$$

where

$$
A_{2}=4 a_{1}^{3}-2\left(2 a_{2}+a_{3}\right) a_{1}{ }^{2}-2\left(a_{2}+2 a_{3}\right) a_{2} a_{1}+4 a_{2}{ }^{2} a_{3},
$$




$$
\begin{aligned}
A_{1}= & \left(8 a_{2}{ }^{2}+20 a_{2} a_{3}-a_{3}{ }^{2}\right) a_{1}{ }^{2}-\left(8 a_{2}{ }^{3}+38 a_{2}{ }^{2} a_{3}+8 a_{2} a_{3}{ }^{2}\right) a_{1} \\
& -a_{2}{ }^{4}+20 a_{2}{ }^{3} a_{3}+8 a_{2}{ }^{2} a_{3}{ }^{2}, \\
A_{0}= & 4 a_{2}\left(a_{1}-a_{2}\right)\left(a_{2}-a_{3}\right)^{3} \neq 0 .
\end{aligned}
$$

We denote this surface by $R_{E}$.

Case (F). $\quad c_{1}+B a_{2} a_{3}=a_{2} a_{3}\left(B-a_{3}\right)$. Then $c_{1}=-a_{2} a_{3}{ }^{2}$ and $c_{2}=-\left(a_{3}-a_{1}\right)^{2}\left(a_{2}\right.$ $\left.-a_{1}\right)$. Further

$$
\left\{\begin{array}{l}
S_{1}=a_{2}+2 a_{3}+y_{0} e^{H}, \quad y_{0}=-\frac{\beta_{1}}{a_{2}\left(a_{2}-a_{1}\right)}, \\
S_{2}=2 a_{2} a_{3}+a_{3}{ }^{2}+a_{1} y_{0} e^{H}, \\
S_{3}=-c_{1}=a_{2} a_{3}{ }^{2} .
\end{array}\right.
$$

In this case the discriminant $\Delta$ of $y^{3}-S_{1} y^{2}+S_{2} y-S_{3}=0$ is

$$
\Delta=y_{0} e^{H}\left(-a_{1}{ }^{2} y_{0}{ }^{3} e^{3 H}+A_{2} y_{0}{ }^{2} e^{2 H}+A_{1} y_{0} e^{H}+A_{0}\right),
$$

where

$$
\begin{aligned}
A_{2}= & 4 a_{1}{ }^{3}-2\left(a_{2}+2 a_{3}\right) a_{1}{ }^{2}-2\left(2 a_{2}+a_{3}\right) a_{3} a_{1}+4 a_{2} a_{3}{ }^{2}, \\
A_{1}= & \left(8 a_{3}{ }^{2}+20 a_{2} a_{3}-a_{2}{ }^{2}\right) a_{1}{ }^{2}-\left(8 a_{3}{ }^{3}+38 a_{3}{ }^{2} a_{2}+8 a_{3} a_{2}{ }^{2}\right) a_{1} \\
& -a_{3}{ }^{4}+20 a_{3}{ }^{3} a_{2}+8 a_{2}{ }^{2} a_{3}{ }^{2}, \\
A_{0}= & -4 a_{3}\left(a_{1}-a_{3}\right)\left(a_{2}-a_{3}\right)^{3} \neq 0 .
\end{aligned}
$$

We denote this surface by $R_{F}$.

The second one is the following simultaneous equation:

$$
\left\{\begin{aligned}
-S_{3} & =\beta_{1} e^{H_{1}}, \\
a_{1}{ }^{3}-S_{1} a_{1}{ }^{2}+S_{2} a_{1}-S_{3} & =c_{1}, \\
a_{2}{ }^{3}-S_{1} a_{2}{ }^{2}+S_{2} a_{2}-S_{3} & =c_{2}, \\
a_{3}{ }^{3}-S_{1} a_{3}{ }^{2}+S_{2} a_{3}-S_{3} & =\beta_{2} e^{H_{2}} .
\end{aligned}\right.
$$

By Borel's unicity theorem we have $H_{1}=H_{2} \equiv H, \beta_{2} a_{1} a_{2}=\beta_{1}\left(a_{3}-a_{1}\right)\left(a_{3}-a_{2}\right)$ and

$$
c_{1} a_{2}\left(a_{3}-a_{2}\right)-c_{2} a_{1}\left(a_{3}-a_{1}\right)=a_{1} a_{2}\left(a_{2}-a_{1}\right)\left(a_{3}-a_{1}\right)\left(a_{3}-a_{2}\right) .
$$

Then

$$
\left\{\begin{array}{l}
S_{1}=\frac{c_{1}}{a_{1}\left(a_{3}-a_{1}\right)}+a_{1}+a_{3}-\frac{\beta_{1} e^{H}}{a_{1} a_{2}}, \\
S_{2}=\frac{c_{1} a_{3}}{a_{1}\left(a_{3}-a_{1}\right)}+a_{1} a_{3}-\frac{\left(a_{1}+a_{2}\right) \beta_{1} e^{H}}{a_{1} a_{2}}, \\
S_{3}=-\beta_{1} e^{H} .
\end{array}\right.
$$


Now we pose the following condition: There is no non-zero constant $B$, being different from $a_{3}$, such that $F(z, B)$ reduces to the form $\alpha e^{X}$, where $\alpha \neq 0$ and $X$ : non-constant entire function. We have

$$
F(z, B)=\frac{\beta_{1} e^{H}}{a_{1} a_{2}}\left(B-a_{1}\right)\left(B-a_{2}\right)+\frac{B\left(B-a_{3}\right)}{a_{1}\left(a_{3}-a_{1}\right)}\left(\left(B-a_{1}\right) a_{1}\left(a_{3}-a_{1}\right)-c_{1}\right) .
$$

Case (G). $\left(B-a_{1}\right) a_{1}\left(a_{3}-a_{1}\right)-c_{1}=\gamma B$ with a non-zero constant $\gamma$, which is independent of $B$. Then $c_{1}=-a_{1}{ }^{2}\left(a_{3}-a_{1}\right)$ and $c_{2}=-a_{8}{ }^{2}\left(a_{3}-a_{8}\right)$. Further

$$
\left\{\begin{array}{l}
S_{1}=a_{3}-\frac{\beta_{1}}{a_{1} a_{2}} e^{H} \equiv a_{3}+y_{0} e^{H}, \\
S_{2}=-\frac{a_{1}+a_{2}}{a_{1} a_{2}} \beta_{1} e^{H} \equiv\left(a_{1}+a_{2}\right) y_{0} e^{H}, \\
S_{3}=-\beta_{1} e^{H} \equiv a_{1} a_{2} y_{0} e^{H} .
\end{array}\right.
$$

Then the discriminant $\Delta$ of $R$ is

$$
\Delta=y_{0} e^{H}\left(A_{3} y_{0}{ }^{3} e^{3 H}+A_{2} y_{0}{ }^{2} e^{2 H}+A_{1} y_{0} e^{H}+A_{0}\right),
$$

where

$$
\begin{aligned}
& A_{3}=4 a_{1} a_{2}-\left(a_{1}+a_{2}\right)^{2}=-\left(a_{1}-a_{2}\right)^{2} \neq 0, \\
& A_{2}=-2\left(a_{1}{ }^{2}-4 a_{1} a_{2}+a_{2}{ }^{2}\right) a_{3}-2\left(a_{1}+a_{2}\right)\left(2 a_{1}-a_{2}\right)\left(a_{1}-2 a_{2}\right) \\
& A_{1}=-\left(a_{1}{ }^{2}-10 a_{1} a_{2}+a_{2}{ }^{2}\right) a_{3}{ }^{2}-18 a_{1} a_{2}\left(a_{1}+a_{2}\right) a_{3}+27 a_{1}{ }^{2} a_{2}{ }^{2}, \\
& A_{0}=4 a_{1} a_{2} a_{3}{ }^{3} \neq 0 .
\end{aligned}
$$

We denote this surface by $R_{G}$.

Case $(\mathrm{H}) . \quad\left(B-a_{1}\right) a_{1}\left(a_{3}-a_{1}\right)-c_{1}=\gamma\left(B-a_{3}\right)$. Then $c_{1}=a_{1}\left(a_{3}-a_{1}\right)^{2}$ and $c_{2}=$ $a_{2}\left(a_{3}-a_{2}\right)^{2}$. Further

$$
\left\{\begin{array}{l}
S_{1}=2 a_{3}+y_{0} e^{H}, \quad y_{0}=\frac{-\beta_{1}}{a_{1} a_{2}}, \\
S_{2}=a_{3}^{2}+\left(a_{1}+a_{2}\right) y_{0} e^{H}, \\
S_{3}=a_{1} a_{2} y_{0} e^{H} .
\end{array}\right.
$$

The discriminant $\Delta$ of $R$ is

$$
\Delta=y_{0} e^{H}\left(A_{3} y_{0}{ }^{3} e^{3 H}+A_{2} y_{0}^{2} e^{2 H}+A_{1} y_{0} e^{H}+A_{0}\right),
$$

where

$$
\begin{aligned}
& A_{3}=-\left(a_{1}-a_{2}\right)^{2} \neq 0 \text {, } \\
& A_{2}=-2 a_{3}{ }^{2}\left(a_{1}+a_{2}\right)-4 a_{3}\left(a_{1}{ }^{2}+4 a_{1} a_{2}+a_{2}{ }^{2}\right) \\
& +2\left(a_{1}+a_{2}\right)\left(2 a_{1}-a_{2}\right)\left(a_{1}-2 a_{2}\right),
\end{aligned}
$$




$$
\begin{aligned}
A_{1}= & -a_{3}{ }^{4}-8 a_{3}{ }^{3}\left(a_{1}+a_{2}\right)+a_{3}{ }^{2}\left(8 a_{1}{ }^{2}+46 a_{1} a_{2}+8 a_{2}{ }^{2}\right) \\
& -36 a_{1} a_{2}\left(a_{1}+a_{2}\right) a_{3}+27 a_{1}{ }^{2} a_{2}{ }^{2}, \\
A_{0}= & -4 a_{3}{ }^{3}\left(a_{3}-a_{1}\right)\left(a_{3}-a_{2}\right) \neq 0 .
\end{aligned}
$$

We denote this surface by $R_{H}$.

\section{§3. Riemann surfaces of $P(R)=6$}

In introduction we have listed up two kinds of Riemann surfaces of six Picard constant. We briefly introduce how to construct them. Let $R$ be the Riemann surface defined by

$$
F(z, y) \equiv y^{3}-S_{1} y^{2}+S_{2} y-S_{3}=0,
$$

where $S_{1}, S_{2}, S_{3}$ are entire functions. Suppose that $P(R)=6$. By Rémoundos' theorem [6] we may consider the following two cases:

$$
\left(\begin{array}{l}
F(z, 0) \\
F\left(z, b_{1}\right) \\
F\left(z, b_{2}\right) \\
F\left(z, b_{3}\right) \\
F\left(z, b_{4}\right)
\end{array}\right)=\left(\begin{array}{c}
c_{1} \\
c_{2} \\
\beta_{1} e^{L_{1}} \\
\beta_{2} e^{L_{2}} \\
\beta_{3} e^{L_{3}}
\end{array}\right), \quad \text { (ii) } \quad=\left(\begin{array}{c}
\beta_{1} e^{L_{1}} \\
c_{1} \\
c_{2} \\
\beta_{2} e^{L_{2}} \\
\beta_{3} e^{L_{3}}
\end{array}\right) .
$$

Here $c_{1}, c_{2}, \beta_{1}, \beta_{2}, \beta_{3}$ are non-zero constants. $L_{3}$ are non-constant entire functions with $L_{j}(0)=0$ for $j=1,2,3$. Further $b_{1}, b_{2}, b_{3}, b_{4}$ are different non-zero complex numbers.

Case (i ). $L_{1}=L_{2}=L_{3}=L$ follows easily. Then

$$
\left\{\begin{array}{l}
S_{1}=x_{0} e^{L}+x_{1}, \\
S_{2}=b_{1} x_{0} e^{L}+x_{2}, \\
S_{3}=x_{3}
\end{array}\right.
$$

with $x_{0}=\beta_{1} / b_{2}\left(b_{1}-b_{2}\right), x_{1}=b_{2}+b_{3}+b_{4}, x_{2}=b_{2} b_{3}+b_{3} b_{4}+b_{2} b_{4}$ and $x_{3}=b_{2} b_{3} b_{4}$. Hence the surface is defined by

$$
y^{3}-\left(x_{0} e^{L}+x_{1}\right) y^{2}+\left(b_{1} x_{0} e^{L}+x_{2}\right) y-x_{3}=0 .
$$

Its discriminant $D$ is

$$
D=-b_{1}{ }^{2} x_{0}{ }^{4} e^{4 L}+\eta_{3} x_{0}{ }^{3} e^{3 L}+\eta_{2} x_{0}{ }^{2} e^{2 L}+\eta_{1} x_{0} e^{L}+\eta_{0},
$$

where

$$
\eta_{3}=4 b_{1}^{3}-2 b_{1}^{2} x_{1}-2 b_{1} x_{2}+4 x_{3},
$$




$$
\begin{aligned}
\eta_{2} & =12 x_{1} x_{3}-18 b_{1} x_{3}-x_{2}{ }^{2}-4 b_{1} x_{1} x_{2}+12 b_{1}{ }^{2} x_{2}-b_{1}{ }^{2} x_{1}{ }^{2}, \\
\eta_{1} & =12 x_{1}{ }^{2} x_{3}-18 b_{1} x_{1} x_{3}-18 x_{2} x_{3}-2 x_{1} x_{2}{ }^{2}+12 b_{1} x_{2}{ }^{2}-2 b_{1} x_{1}{ }^{2} x_{2}, \\
\eta_{0} & =4 x_{1}{ }^{3} x_{3}-x_{1}{ }^{2} x_{2}{ }^{2}+27 x_{3}{ }^{2}-18 x_{1} x_{2} x_{3}+4 x_{2}{ }^{3} \\
& =-\left(b_{2}-b_{3}\right)^{2}\left(b_{2}-b_{4}\right)^{2}\left(b_{3}-b_{4}\right)^{2} \neq 0 .
\end{aligned}
$$

This surface is denoted by $X_{1}$.

Case (ii). $L_{1}=L_{2}=L_{3}=L$ follows easily. Then

$$
\left\{\begin{array}{l}
S_{1}=x_{0} e^{L}+x_{1}, \\
S_{2}=\left(b_{1}+b_{2}\right) x_{0} e^{L}+x_{2}, \\
S_{3}=b_{1} b_{2} x_{0} e^{L}
\end{array}\right.
$$

with $x_{0}=-\beta_{1} / b_{1} b_{2}, x_{1}=b_{3}+b_{4}, x_{2}=b_{3} b_{4}$. Hence the surface is defined by

$$
y^{3}-\left(x_{0} e^{L}+x_{1}\right) y^{2}+\left\{\left(b_{1}+b_{2}\right) x_{0} e^{L}+x_{2}\right\} y-b_{1} b_{2} x_{0} e^{L}=0 .
$$

Its discriminant $D$ is

$$
D=\left(b_{1}-b_{2}\right)^{2} x_{0}^{4} e^{4 L}+\eta_{3} x_{0}^{3} e^{3 L}+\eta_{2} x_{0}^{2} e^{2 L}+\eta_{1} x_{0} e^{L}+\eta_{0},
$$

where

$$
\begin{aligned}
\eta_{3}= & \left(2 b_{1}{ }^{2}-8 b_{1} b_{2}+2 b_{2}{ }^{2}\right) x_{1}+2\left(b_{1}+b_{2}\right) x_{2} \\
& -2\left(b_{1}+b_{2}\right)\left(2 b_{1}{ }^{2}-5 b_{1} b_{2}+2 b_{2}{ }^{2}\right), \\
\eta_{2}= & \left(b_{1}{ }^{2}-10 b_{1} b_{2}+b_{2}{ }^{2}\right) x_{1}{ }^{2}+4\left(b_{1}+b_{2}\right) x_{1} x_{2}+x_{2}{ }^{2} \\
& +18\left(b_{1}+b_{2}\right) b_{1} b_{2} x_{1}-\left(12 b_{1}{ }^{2}+6 b_{1} b_{2}+12 b_{2}{ }^{2}\right) x_{2}-27 b_{1}{ }^{2} b_{2}{ }^{2}, \\
\eta_{1}= & -4 b_{1} b_{2} x_{1}{ }^{3}+2\left(b_{1}+b_{2}\right) x_{1}{ }^{2} x_{2}+2 x_{1} x_{2}{ }^{2}+18 b_{1} b_{2} x_{1} x_{2} \\
& -12\left(b_{1}+b_{2}\right) x_{2}{ }^{2}, \\
\eta_{0}= & x_{1}{ }^{2} x_{2}{ }^{2}-4 x_{2}{ }^{3}=b_{3}{ }^{2} b_{4}{ }^{2}\left(b_{3}-b_{4}\right)^{2} \neq 0 .
\end{aligned}
$$

This surface is denoted by $X_{2}$.

\section{§4. A lemma}

It is necessary to give an explicit proof of the following.

Lemma. Let $R$ be the Riemann surface $R_{A}$ defined by

$$
y^{3}-S_{1} y^{2}+S_{2} y-S_{3}=0
$$

with $S_{1}=x_{1}, S_{2}=x_{0} e^{H}+x_{2}, S_{3}=x_{3}$, where $x_{0}, x_{1}, x_{2}$ and $x_{3}$ are constants, $x_{0} \neq 0$, 
$x_{1}=a_{2}+a_{3}+a_{4}, x_{2}=a_{2} a_{3}+a_{3} a_{4}+a_{2} a_{4}, x_{3}=a_{2} a_{3} a_{4}$. Let $F$ be a regular function on $R_{A}$. Then $F$ is representable as

$$
F=f_{1}+f_{2} y+f_{3} y^{2},
$$

where $f_{1}, f_{2}$ and $f_{3}$ are meromorphic functions in $|z|<\infty$, all of which are regular at any points $z$ satisfying $H^{\prime}(z) \neq 0$.

Proof. Let $z_{0}$ be a point satisfying $H^{\prime}\left(z_{0}\right) \neq 0$.

Case 1). There are two different points of $R_{A}$ over $z_{0}$. Of course one is a branch point and the other is an ordinary point. Then $y$ has two branches $y_{1}$ and $y_{2}$ for which

$$
y_{1}=A_{0}+A_{1}\left(z-z_{0}\right)^{p / 2}+A_{2}\left(z-z_{0}\right)^{(p+1 / 2)}+\cdots
$$

with $A_{0} A_{1} \neq 0$ and

$$
y_{2}=B_{0}+B_{1}\left(z-z_{0}\right)^{q}+B_{2}\left(z-z_{0}\right)^{q+1}+\cdots
$$

with $B_{0} B_{1} \neq 0 . \quad A_{0} B_{0} \neq 0$, since $y$ does not vanish. If $p \geqq 3$, then $y_{1}{ }^{3}-x_{1} y_{1}{ }^{2}+$ $\left(x_{0} e^{H(z)}+x_{2}\right) y_{1}-x_{3}=0$ gives

$$
\begin{aligned}
& A_{0}{ }^{3}+3 A_{0}{ }^{2} A_{1}\left(z-z_{0}\right)^{p / 2}+\cdots-x_{1}\left(A_{0}{ }^{2}+2 A_{0} A_{1}\left(z-z_{0}\right)^{p / 2}+\cdots\right) \\
& +\left[x_{0} e^{H\left(z_{0}\right)}\left\{1+\varepsilon_{1}\left(z-z_{0}\right)+\cdots\right\}+x_{2}\right]\left(A_{0}+A_{1}\left(z-z_{0}\right)^{p / 2}+\cdots\right)-x_{3}=0
\end{aligned}
$$

with $\varepsilon_{1} \neq 0$. This gives $\varepsilon_{1} x_{0} e^{H\left(z_{0}\right)} A_{0}=0$, which is absurd. If $p=2$, then there is the smallest index $s$ for which

$$
y_{1}=A_{0}+A_{1}\left(z-z_{0}\right)+\cdots+A_{s}^{*}\left(z-z_{0}\right)^{s / 2}+\cdots
$$

with an odd $s$ and a non-zero constant $A_{s}{ }^{*}$. Then we have

$$
\begin{aligned}
A_{0}{ }^{3}+ & 3{A_{0}}^{2} A_{1}\left(z-z_{0}\right)+\cdots+3 A_{0}{ }^{2} A_{s} *\left(z-z_{0}\right)^{s / 2}+\cdots \\
& -x_{1}\left(A_{0}{ }^{2}+2 A_{0} A_{1}\left(z-z_{0}\right)+\cdots+2 A_{0} A_{s}{ }^{*}\left(z-z_{0}\right)^{s / 2}+\cdots\right) \\
& +\left[x_{0} e^{H\left(z_{0}\right)}\left\{1+\varepsilon_{1}\left(z-z_{0}\right)+\cdots\right\}+x_{2}\right]\left(A_{0}+A_{1}\left(z-z_{0}\right)+\cdots+A_{s} *\left(z-z_{0}\right)^{s / 2}\right. \\
& +\cdots-x_{3}=0 .
\end{aligned}
$$

Hence from the coefficient of $\left(z-z_{0}\right)^{s / 2}$,

which gives

$$
\left\{3 A_{0}{ }^{2}-2 x_{1} A_{0}+x_{0} e^{H\left(z_{0}\right)}+x_{2}\right\} A_{s}^{*}=0,
$$

$$
3 A_{0}{ }^{2}-2 x_{1} A_{0}+x_{0} e^{H\left(z_{0}\right)}+x_{2}=0 .
$$

The coefficient of $z-z_{0}$ is

$$
\left\{3 A_{0}{ }^{2}-2 x_{1} A_{0}+x_{0} e^{H\left(z_{0}\right)}+x_{2}\right\} A_{1}+x_{0} e^{H\left(z_{0}\right)} \varepsilon_{1} A_{0}=0 .
$$


Hence $x_{0} e^{H\left(\varepsilon_{0}\right)} \varepsilon_{1} A_{0}=0$, which is absurd. Hence

$$
y_{1}=A_{0}+A_{1}\left(z-z_{0}\right)^{1 / 2}+A_{2}\left(z-z_{0}\right)+\cdots .
$$

Then from the coefficient of $\left(z-z_{0}\right)^{1 / 2}$ of $y_{1}{ }^{3}-x_{1} y_{1}{ }^{2}+\left(x_{0} e^{H(z)}+x_{2}\right) y_{1}-x_{3}=0$.

$$
\left\{3 A_{0}^{2}-2 x_{1} A_{0}+x_{0} e^{H\left(z_{0}\right)}+x_{2}\right\} A_{1}=0 \text {. }
$$

Hence

$$
3 A_{0}{ }^{2}-2 x_{1} A_{0}+x_{0} e^{H\left(z_{0}\right)}+x_{2}=0 .
$$

We shall make use of this relation later.

Similarly for the one-valued branch $y_{2}$ we have

$$
y_{2}=B_{0}+B_{1}\left(z-z_{0}\right)+\cdots .
$$

Assume that $F_{1}=f_{1}+f_{2} y_{1}+f_{3} y_{1}{ }^{2}$ and $F_{2}=f_{1}+f_{2} y_{2}+f_{3} y_{2}{ }^{2}$ are pole-free at $z_{0}$. Then put

$$
\begin{aligned}
& f_{1}=\frac{\alpha_{n}}{\left(z-z_{0}\right)^{n}}+\frac{\alpha_{n-1}}{\left(z-z_{0}\right)^{n-1}}+\cdots, \\
& f_{2}=\frac{\beta_{n}}{\left(z-z_{0}\right)^{n}}+\frac{\beta_{n-1}}{\left(z-z_{0}\right)^{n-1}}+\cdots, \\
& f_{3}=\frac{\gamma_{n}}{\left(z-z_{0}\right)^{n}}+\frac{\gamma_{n-1}}{\left(z-z_{0}\right)^{n-1}}+\cdots .
\end{aligned}
$$

with $\left(\alpha_{n}, \beta_{n}, \gamma_{n}\right) \neq(0,0,0)$.

Then we have

$$
\begin{aligned}
F_{1}= & f_{1}+f_{2} y_{1}+f_{3} y_{1}{ }^{2} \\
= & \frac{\alpha_{n}}{\left(z-z_{0}\right)^{n}}+\frac{\alpha_{n-1}}{\left(z-z_{0}\right)^{n-1}}+\cdots \\
& +\left\{\frac{\beta_{n}}{\left(z-z_{0}\right)^{n}}+\frac{\beta_{n-1}}{\left(z-z_{0}\right)^{n-1}}+\cdots\right\}\left\{A_{0}+A_{1}\left(z-z_{0}\right)^{1 / 2}+A_{2}\left(z-z_{0}\right)+\cdots\right\} \\
& +\left\{\frac{\gamma_{n}}{\left(z-z_{0}\right)^{n}}+\frac{\gamma_{n-1}}{\left(z-z_{0}\right)^{n-1}}+\cdots\right\}\left\{A_{0}{ }^{2}+2 A_{0} A_{1}\left(z-z_{0}\right)^{1 / 2}\right. \\
& \left.+\left(A_{1}{ }^{2}+2 A_{0} A_{2}\right)\left(z-z_{0}\right)+\cdots\right\} .
\end{aligned}
$$

Then

$$
\begin{aligned}
& \alpha_{n}+\beta_{n} A_{0}+\gamma_{n} A_{0}{ }^{2}=0, \\
& \beta_{n} A_{1}+\gamma_{n} 2 A_{0} A_{1}=0 .
\end{aligned}
$$

Similarly for $F_{2}=f_{1}+f_{2} y_{2}+f_{3} y_{2}^{2}$ we have

$$
\alpha_{n}+\beta_{n} B_{0}+\gamma_{n} B_{0}^{2}=0 \text {. }
$$


Hence $\left\{\beta_{n}+\gamma_{n}\left(A_{0}+B_{0}\right)\right\}\left(A_{0}-B_{0}\right)=0$. If $A_{0} \neq B_{0}$, then $\beta_{n}+\gamma_{n}\left(A_{0}+B_{0}\right)=0$. On the other hand we have $\beta_{n}+2 \gamma_{n} A_{0}=0$. And if $\gamma_{n} \neq 0$, we have $A_{0}=B_{0}$, which is absurd. If $\gamma_{n}=0$ then we have $\beta_{n}=\alpha_{n}=0$, which is absurd. Therefore $A_{0}$ $=B_{0}$. By $y_{2}{ }^{3}-x_{1} y_{2}{ }^{2}+\left(x_{0} e^{H(z)}+x_{2}\right) y_{2}-x_{3}=0$ we have

$$
\left\{3 A_{0}^{2}-2 x_{1} A_{0}+x_{0} e^{H\left(z_{0}\right)}+x_{2}\right\} B_{1}+x_{0} e^{H\left(z_{0}\right)} \varepsilon_{1} A_{0}=0 .
$$

Hence we have an absurdity relation $x_{0} e^{H\left(z_{0}\right)} \varepsilon_{1} A_{0}=0$.

Case 2). There is only one point of $R_{A}$ over $z_{0}$. Then

$$
y(z)=A_{0}+A_{p}\left(z-z_{0}\right)^{p / 3}+\cdots .
$$

If $p \geqq 4$, then the coefficient of $z-z_{0}$ of $y^{3}-x_{1} y^{2}+\left(x_{0} e^{H(z)}+x_{2}\right) y-x_{3}=0$ is equal to $x_{0} e^{H\left(z_{0}\right)} \varepsilon_{1} A_{0}$. Hence this vanishes, which is impossible. If $p=3$, then there is the smallest index $s$ for which

$$
y=A_{0}+A_{3}\left(z-z_{0}\right)+\cdots+A_{s} *\left(z-z_{0}\right)^{s / 3}+\cdots
$$

with $s \neq 0 \bmod 3$ and non-zero $A_{s} *$. Then the coefficient of $\left(z-z_{0}\right)^{s / 3}$ in the Puisseux expansion of $y^{3}-x_{1} y^{2}+\left(x_{0} e^{H(z)}+x_{2}\right) y-x_{3}=0$ is equal to

Hence

$$
3 A_{0}^{2} A_{s}^{*}-2 x_{1} A_{0} A_{s}^{*}+\left(x_{0} e^{H\left(z_{0}\right)}+x_{2}\right) A_{s}^{*}=0 .
$$

$$
3 A_{0}{ }^{2}-2 x_{1} A_{0}+x_{0} e^{H\left(z_{0}\right)}+x_{2}=0 .
$$

On the other hand the coefficient of $z-z_{0}$ is equal to

$$
\left(3 A_{0}^{2}-2 x_{1} A_{0}+x_{0} e^{H\left(z_{0}\right)}+x_{2}\right) A_{1}+x_{0} e^{H\left(z_{0}\right)} \varepsilon_{1} A_{0}=0 .
$$

This is evidently impossible. Therefore $p=2$ or $p=1$.

Suppose that $p=1$ and further that $y=A_{0}+A_{1}\left(z-z_{0}\right)^{1 / 3}+A_{3}\left(z-z_{0}\right)+A_{4}(z-$ $\left.z_{0}\right)^{4 / 3}+\cdots$ with $A_{1} \neq 0$. Then

$$
\begin{aligned}
F= & f_{1}+f_{2} y+f_{3} y^{2} \\
= & \frac{\alpha_{n}}{\left(z-z_{0}\right)^{n}}+\frac{\alpha_{n-1}}{\left(z-z_{0}\right)^{n-1}}+\cdots \\
& +\left\{\frac{\beta_{n}}{\left(z-z_{0}\right)^{n}}+\frac{\beta_{n-1}}{\left(z-z_{0}\right)^{n-1}}+\cdots\right\}\left\{A_{0}+A_{1}\left(z-z_{0}\right)^{1 / 3}+A_{3}\left(z-z_{0}\right)+\cdots\right\} \\
& +\left\{\frac{\gamma_{n}}{\left(z-z_{0}\right)^{n}}+\frac{\gamma_{n-1}}{\left(z-z_{0}\right)^{n-1}}+\cdots\right\}\left\{A_{0}{ }^{2}+2 A_{0} A_{1}\left(z-z_{0}\right)^{1 / 3}+A_{1}{ }^{2}\left(z-z_{0}\right)^{2 / 3}+\cdots\right\} .
\end{aligned}
$$

Since $F$ is pole-free at $z_{0}$,

and

$$
\begin{aligned}
& \alpha_{n}+\beta_{n} A_{0}+\gamma_{n} A_{0}{ }^{2}=0, \\
& \beta_{n} A_{1}+2 \gamma_{n} A_{0} A_{1}=0 .
\end{aligned}
$$

$$
\gamma_{n} A_{1}^{2}=0 \text {. }
$$


Then $\gamma_{n}=0$ implies $\beta_{n}=0$ and $\alpha_{n}=0$. This holds for all $n \geqq 1$. Hence we arrive at a contradiction.

Suppose that $p=1$ and further that $y=A_{0}+A_{1}\left(z-z_{0}\right)^{1 / 3}+A_{2}\left(z-z_{0}\right)^{2 / 3}+A_{3}(z-$ $\left.z_{0}\right)+\cdots$ with $A_{1} A_{2} \neq 0$. Similarly we have

and

$$
\begin{aligned}
& \alpha_{n}+\beta_{n} A_{0}+\gamma_{n} A_{0}{ }^{2}=0, \\
& \left(\beta_{n}+2 \gamma_{n} A_{0}\right) A_{1}=0
\end{aligned}
$$

$$
\beta_{n} A_{2}+\gamma_{n}\left(2 A_{0} A_{2}+A_{1}^{2}\right)=0 \text {. }
$$

These relations contain a contradiction similarly.

Suppose that $p=2$. Then $y=A_{0}+A_{2}\left(z-z_{0}\right)^{2 / 3}+A_{3}\left(z-z_{0}\right)+\cdots, A_{0} A_{2} \neq 0$. In this case

$$
\begin{aligned}
0= & y^{3}-x_{1} y^{2}+\left(x_{0} e^{H(z)}+x_{2}\right) y-x_{3} \\
= & A_{0}{ }^{3}+3 A_{0}{ }^{2} A_{2}\left(z-z_{0}\right)^{2 / 3}+3 A_{0}{ }^{2} A_{3}\left(z-z_{0}\right)+\cdots \\
& -x_{1}\left(A_{0}{ }^{2}+2 A_{0} A_{2}\left(z-z_{0}\right)^{2 / 3}+2 A_{0} A_{3}\left(z-z_{0}\right)+\cdots\right) \\
& +\left\{x_{0} e^{H\left(z_{0}\right)}\left(1+\varepsilon_{1}\left(z-z_{0}\right)+\cdots\right)+x_{2}\right\}\left(A_{0}+A_{2}\left(z-z_{0}\right)^{2 / 3}+A_{3}\left(z-z_{0}\right)+\cdots\right)-x_{3} .
\end{aligned}
$$

Hence we have

and

$$
\left(3 A_{0}^{2}-2 x_{1} A_{0}+x_{0} e^{H\left(z_{0}\right)}+x_{2}\right) A_{2}=0
$$

$$
\left(3 A_{0}^{2}-2 x_{1} A_{0}+x_{0} e^{H\left(z_{0}\right)}+x_{2}\right) A_{3}+x_{0} e^{H\left(z_{0}\right)} \varepsilon_{1} A_{0}=0 .
$$

Therefore we have a contradiction.

Case 3). There are three ordinary points of $R_{A}$ over $z_{0}$. Then there are three different branches of $y$ around these points. Suppose that

$$
y_{1}=A_{0}+A_{1}\left(z-z_{0}\right)^{p}+\cdots
$$

with $p \geqq 2, A_{0} A_{1} \neq 0$. Then by $y_{1}^{3}-x_{1} y_{1}^{2}+\left(x_{0} e^{H(z)}+x_{2}\right) y_{1}-x_{3}=0$ we have $x_{0} e^{H\left(z_{0}\right)} \varepsilon_{1} A_{0}=0$, which is absurd. Hence $y_{1}=A_{0}+A_{1}\left(z-z_{0}\right)+A_{2}\left(z-z_{0}\right)^{2}+\cdots$. Similarly

and

$$
y_{2}=B_{0}+B_{2}\left(z-z_{0}\right)+B_{2}\left(z-z_{0}\right)^{2}+\cdots, \quad B_{0} B_{1} \neq 0
$$

$$
y_{3}=C_{0}+C_{1}\left(z-z_{0}\right)+C_{2}\left(z-z_{0}\right)^{2}+\cdots, \quad C_{0} C_{1} \neq 0 \text {. }
$$

Let us put

$$
\begin{aligned}
& f_{1}=\frac{\alpha_{n}}{\left(z-z_{0}\right)^{n}}+\frac{\alpha_{n-1}}{\left(z-z_{0}\right)^{n-1}}+\cdots, \\
& f_{2}=\frac{\beta_{n}}{\left(z-z_{0}\right)^{n}}+\frac{\beta_{n-1}}{\left(z-z_{0}\right)^{n-1}}+\cdots,
\end{aligned}
$$




$$
f_{3}=\frac{\gamma_{n}}{\left(z-z_{0}\right)^{n}}+\frac{\gamma_{n-1}}{\left(z-z_{0}\right)^{n-1}}+\cdots
$$

Then $F=f_{1}+f_{2} y+f_{3} y^{2}$ should be pole-free at $z_{0}$ for any branch of $y$. Hence

Then

$$
\begin{aligned}
& \alpha_{n}+\beta_{n} A_{0}+\gamma_{n} A_{0}^{2}=0, \\
& \alpha_{n}+\beta_{n} B_{0}+\gamma_{n} B_{0}^{2}=0, \\
& \alpha_{n}+\beta_{n} C_{0}+\gamma_{n} C_{0}{ }^{2}=0 .
\end{aligned}
$$

and

$$
\left(\beta_{n}+\gamma_{n}\left(A_{0}+B_{0}\right)\right)\left(A_{0}-B_{0}\right)=0
$$

$$
\left(\beta_{n}+\gamma_{n}\left(A_{0}+C_{0}\right)\right)\left(A_{0}-C_{0}\right)=0 \text {. }
$$

If $A_{0} \neq B_{0}$ and $A_{0} \neq C_{0}$, then $\beta_{n}+\gamma_{n}\left(A_{0}+B_{0}\right)=\beta_{n}+\gamma_{n}\left(A_{0}+C_{0}\right)=0$. Hence $\gamma_{n}\left(B_{0}-\right.$ $\left.C_{0}\right)=0$. If $B_{0} \neq C_{0}$, then $\gamma_{n}=0$ and $\beta_{n}=0, \alpha_{n}=0$. This gives a contradiction. Hence $B_{0}=C_{0}$. Therefore we have either $A_{0}=B_{0}$ or $A_{0}=C_{0}$ or $B_{0}=C_{0}$. Suppose now $A_{0}=B_{0}$.

Then by $y_{1}^{3}-x_{1} y_{1}{ }^{2}+\left(x_{0} e^{H(z)}+x_{2}\right) y_{1}-x_{3}=0$ we have

$$
\begin{gathered}
A_{0}{ }^{3}-x_{1} A_{0}{ }^{2}+\left(x_{0} e^{H\left(z_{0}\right)}+x_{2}\right) A_{0}-x_{3}=0, \\
\left(3 A_{0}{ }^{2}-2 x_{1} A_{0}+x_{0} e^{H\left(z_{0}\right)}+x_{2}\right) A_{1}+x_{0} e^{H\left(z_{0}\right)} \varepsilon_{1} A_{0}=0 .
\end{gathered}
$$

Similarly for $y_{2}$ we have

$$
\left(3 B_{0}^{2}-2 x_{1} B_{0}+x_{0} e^{H\left(z_{0}\right)}+x_{2}\right) B_{1}+x_{0} e^{H\left(z_{0}\right)} \varepsilon_{1} B_{0}=0 .
$$

By $A_{0}=B_{0}$ we have

$$
\left(3 A_{0}{ }^{2}-2 x_{1} A_{0}+x_{0} e^{H\left(z_{0}\right)}+x_{2}\right)\left(A_{1}-B_{1}\right)=0 .
$$

Suppose that $A_{1} \neq B_{1}$. Then $3 A_{0}{ }^{2}-2 x_{1} A_{0}+x_{0} e^{H\left(z_{0}\right)}+x_{2}=0$, whence follows $x_{0} e^{H\left(z_{0}\right)} \varepsilon_{1} A_{0}=0$, which is impossible. Hence $A_{1}=B_{1}$. In general

$$
\left\{3 A_{0}{ }^{2}-2 x_{1} A_{0}+x_{0} e^{H\left(z_{0}\right)}+x_{2}\right\} A_{m}+P_{m}\left(A_{0}, A_{1}, \cdots, A_{m-1}, \varepsilon_{1}, \varepsilon_{2}, \cdots, \varepsilon_{m}\right)=0
$$

and

$$
\left\{3 A_{0}{ }^{2}-2 x_{1} A_{0}+x_{0} e^{H\left(z_{0}\right)}+x_{2}\right\} B_{m}+P_{m}\left(A_{0}, A_{1}, \cdots, A_{m-1}, \varepsilon_{1}, \varepsilon_{2}, \cdots, \varepsilon_{m}\right)=0,
$$

if $A_{0}=B_{0}, A_{1}=B_{1}, \cdots, A_{m-1}=B_{m-1}$, where $\varepsilon_{\jmath}, j=1, \cdots, m$ are defined by

$$
x_{0} e^{H\left(z_{0}\right)}+x_{2}=x_{0} e^{H\left(z_{0}\right)}+x_{2}+x_{0} e^{H\left(z_{0}\right)} \sum_{j=1}^{\infty} \varepsilon_{j}\left(z-z_{0}\right)^{j} .
$$

Since $3 A_{0}{ }^{2}-2 x_{1} A_{0}+x_{0} e^{H\left(z_{0}\right)}+x_{2} \neq 0$, we have $A_{m}=B_{m}$. Thus we have $y_{1} \equiv y_{2}$, which is absurd.

Similar lemma hold for the surfaces $X_{1}, R_{B}$ and $R_{E}$. Proofs are quite similar. Further it is sufficient to prove Lemma for the surfaces $R_{A}, R_{B}$ and 
$R_{E}$. In $\S 7$ we show that, when $e^{H}$ is commonly appeared, $R_{D} \sim R_{A}, R_{C} \sim R_{B}$ and $R_{F} \sim R_{G} \sim R_{H} \sim R_{E}$, where $\sim$ means the conformal equivalence by a suitable linear transformation $Y=\alpha y+\beta$. Evidently $X_{1} \sim X_{2}$ too, if $e^{L}$ is common.

\section{§. Transformation formula of discriminants}

Let $R$ be the surface $R_{A}: y^{3}-S_{1} y^{2}+S_{2} y-S_{3}=0$ with $S_{1}=y_{1}, S_{2}=y_{0} e^{H}+y_{2}$, $S_{3}=y_{3}$, where $y_{0}$ is a non-zero constant and $y_{1}=a_{2}+a_{3}+a_{4}, y_{2}=a_{2} a_{3}+a_{3} a_{4}+$ $a_{2} a_{4}, y_{3}=a_{2} a_{3} a_{4}$ and $H$ is an entire function.

From now on we shall assume that the surface is of finite order, that is,

$$
H \text { is a polynomial. }
$$

The same assumption holds in $\S 6$ too.

Now suppose that $P(R)=6$. Then there exists an entire function $f$ on $R$ with $P(f)=6$. We can make use of Lemma in $\S 4$. Then $f$ is representable as

as in Lemma.

$$
f=f_{1}+f_{2} y+f_{3} y^{2}
$$

For simplicity's sake we put $F=f_{1}-f$. Then

$$
\begin{gathered}
F+f_{2} y+f_{3} y^{2}=0, \\
f_{3} S_{3}+\left(F-f_{3} S_{2}\right) y+\left(f_{2}+f_{3} S_{1}\right) y^{2}=0, \\
\left(f_{2}+f_{3} S_{1}\right) S_{3}+\left(f_{3} S_{3}-f_{3} S_{1} S_{2}-f_{2} S_{2}\right) y+\left(F+f_{2} S_{1}+f_{3}\left(S_{1}{ }^{2}-S_{2}\right)\right) y^{2}=0 .
\end{gathered}
$$

By eliminating $y$ and $y^{2}$ we have

$$
F^{3}+Y_{0} F^{2}+Y_{1} F+Y_{2}=0 \text {, }
$$

where

$$
\left\{\begin{array}{l}
Y_{0}=f_{2} S_{1}+f_{3}\left(S_{1}{ }^{2}-2 S_{2}\right), \\
Y_{1}=f_{2}{ }^{2} S_{2}+f_{2} f_{3}\left(S_{1} S_{2}-3 S_{3}\right)+f_{3}{ }^{2}\left(S_{2}{ }^{2}-2 S_{1} S_{3}\right), \\
Y_{2}=f_{2}{ }^{3} S_{3}+f_{2}{ }^{2} f_{3} S_{1} S_{3}+f_{2} f_{3}{ }^{2} S_{2} S_{3}+f_{3}{ }^{3} S_{3}{ }^{2} .
\end{array}\right.
$$

This gives

$$
f^{3}-f^{2} U_{1}+f U_{2}-U_{3}=0
$$

with

$$
\left\{\begin{array}{l}
U_{1}=3 f_{1}+Y_{0}, \\
U_{2}=3 f_{1}^{2}+2 f_{1} Y_{0}+Y_{1}, \\
U_{3}=f_{1}^{3}+f_{1}^{2} Y_{0}+f_{1} Y_{1}+Y_{2},
\end{array}\right.
$$

$U_{1}, U_{2}$ and $U_{2}$ are all entire, since $f$ is a three-valued entire algebroid function. Let $g$ be $f-U_{1} / 3$. Then $g^{3}+A g+B=0$ with 


$$
\begin{aligned}
& A=\frac{1}{3}\left(-U_{1}{ }^{2}+3 U_{2}\right), \\
& B=\frac{1}{27}\left(-2 U_{1}{ }^{3}+9 U_{1} U_{2}-27 U_{3}\right) .
\end{aligned}
$$

Then the discriminant $D$ is equal to $4 A^{3}+27 B^{2}$. Hence

$$
D=4 U_{1}{ }^{3} U_{3}-U_{1}{ }^{2} U_{2}{ }^{2}-18 U_{1} U_{2} U_{3}+4 U_{2}{ }^{3}+27 U_{3}{ }^{2} .
$$

For simplicity's sake we put

and

$$
\begin{gathered}
A=\frac{1}{3}\left(\alpha_{1} f_{2}^{2}+\alpha_{2} f_{2} f_{3}+\alpha_{3} f_{3}{ }^{2}\right), \\
\left\{\begin{array}{l}
\alpha_{1}=3 S_{2}-S_{1}^{2} \\
\alpha_{2}=-2 S_{1}^{3}+7 S_{1} S_{2}-9 S_{3} \\
\alpha_{3}=-S_{1}{ }^{4}+4 S_{1}{ }^{2} S_{2}-6 S_{1} S_{3}-S_{2}{ }^{2}
\end{array}\right.
\end{gathered}
$$

$$
\begin{gathered}
B=\frac{1}{27}\left(\beta_{1} f_{2}{ }^{3}+\beta_{2} f_{2}{ }^{2} f_{3}+\beta_{3} f_{2} f_{3}{ }^{2}+\beta_{4} f_{3}{ }^{3}\right) \\
\left\{\begin{array}{l}
\beta_{1}=-2 S_{1}{ }^{3}+9 S_{1} S_{2}-27 S_{3} \\
\beta_{2}=-6 S_{1}{ }^{4}+30 S_{1}{ }^{2} S_{2}-54 S_{1} S_{3}-18 S_{2}{ }^{2} \\
\beta_{3}=-6 S_{1}{ }^{5}+33 S_{1}{ }^{3} S_{2}-45 S_{1}{ }^{2} S_{3}-33 S_{1} S_{2}{ }^{2}+27 S_{2} S_{3}, \\
\beta_{4}=-2 S_{1}{ }^{6}+12 S_{1}{ }^{4} S_{2}-18 S_{1}{ }^{3} S_{3}-15 S_{1}{ }^{2} S_{2}{ }^{2}+36 S_{1} S_{2} S_{3}-2 S_{2}{ }^{3}-27 S_{3}{ }^{2}
\end{array}\right.
\end{gathered}
$$

Then

$$
\begin{aligned}
& D=4 A^{3}+27 B^{2}= \frac{1}{27}\left\{f_{2}{ }^{6}\left(4 \alpha_{1}{ }^{3}+\beta_{1}{ }^{2}\right)+f_{2}{ }^{5} f_{3}\left(12 \alpha_{1}{ }^{2} \alpha_{2}+2 \beta_{1} \beta_{2}\right)\right. \\
&+f_{2}{ }^{4} f_{3}{ }^{2}\left(12 \alpha_{1}{ }^{2} \alpha_{3}+12 \alpha_{1} \alpha_{2}{ }^{2}+2 \beta_{1} \beta_{3}+\beta_{2}{ }^{2}\right) \\
&+f_{2}{ }^{3} f_{3}{ }^{3}\left(24 \alpha_{1} \alpha_{2} \alpha_{3}+4 \alpha_{2}{ }^{3}+2 \beta_{1} \beta_{4}+2 \beta_{2} \beta_{3}\right) \\
&+f_{2}{ }^{2} f_{3}{ }^{4}\left(12 \alpha_{1} \alpha_{3}{ }^{2}+12 \alpha_{2}{ }^{2} \alpha_{3}+2 \beta_{2} \beta_{4}+\beta_{3}{ }^{2}\right) \\
&\left.+f_{2} f_{3}{ }^{5}\left(12 \alpha_{2} \alpha_{3}{ }^{2}+2 \beta_{3} \beta_{4}\right)+f_{3}{ }^{6}\left(4 \alpha_{3}{ }^{3}+\beta_{4}{ }^{2}\right)\right\} \\
&=\Delta\left\{f_{2}{ }^{6}+4 S_{1} f_{2}{ }^{5} f_{3}+2\left(3 S_{1}{ }^{2}+S_{2}\right) f_{2}{ }^{4} f_{3}{ }^{2}+\left(4 S_{1}{ }^{3}+6 S_{1} S_{2}-2 S_{3}\right) f_{2}{ }^{3} f_{3}{ }^{3}\right. \\
&+\left(S_{1}{ }^{4}+6 S_{1}{ }^{2} S_{2}-4 S_{1} S_{3}+S_{2}{ }^{2}\right) f_{2}{ }^{2} f_{3}{ }^{4}+2\left(S_{1}{ }^{3} S_{2}-S_{1}{ }^{2} S_{3}+S_{1} S_{2}{ }^{2}-S_{2} S_{3}\right) f_{2} f_{3}{ }^{5} \\
&+\left(S_{1}{ }^{2} S_{2}{ }^{2}-2 S_{1} S_{2} S_{3}+\right.\left.\left.S_{3}{ }^{2}\right) f_{3}{ }^{6}\right\} \\
&=\Delta\left\{f_{2}{ }^{3}+2 S_{1} f_{2}{ }^{2} f_{3}+(\right.\left.\left.S_{1}{ }^{2}+S_{2}\right) f_{2} f_{3}{ }^{2}+\left(S_{1} S_{2}-S_{3}\right) f_{3}{ }^{3}\right\}^{2},
\end{aligned}
$$


where $\Delta$ is the discriminant of $y^{3}-S_{1} y^{2}+S_{2} y-S_{3}=0$, that is,

$$
\begin{aligned}
\Delta & =\frac{4}{27} \alpha_{1}{ }^{3}+\frac{1}{27}{\beta_{1}{ }^{2}} \\
& =4 S_{1}{ }^{3} S_{3}-S_{1}{ }^{2}{S_{2}}^{2}-18 S_{1} S_{2} S_{3}+4{S_{2}}^{3}+27 S_{3}{ }^{2} .
\end{aligned}
$$

Let us put the above formula as

$$
D=\Delta \cdot G^{2} .
$$

$G$ may have poles at most at zeros of $H^{\prime}$.

We need more precise result on $D=\Delta \cdot G^{2}$. Evidently the poles of $G$ are finite in number. Let us put

$$
D=-b_{1}^{2}\left(x_{0} e^{L}-\gamma_{1}\right)\left(x_{0} e^{L}-\gamma_{2}\right)\left(x_{0} e^{L}-\gamma_{3}\right)\left(x_{0} e^{L}-\gamma_{4}\right)
$$

and

$$
\Delta=4\left(y_{0} e^{H}-\delta_{1}\right)\left(y_{0} e^{H}-\delta_{2}\right)\left(y_{0} e^{H}-\delta_{3}\right) .
$$

Case 1). The counting function of simple zeros of $\Delta$ satisfies

$$
N_{2}(r, 0, \Delta) \sim 3 T\left(r, e^{H}\right),
$$

that is, $\delta_{i} \neq \delta_{l}$ for $i \neq l$. Then

$$
N_{2}(r, 0, \Delta)=N_{2}(r, 0, D) \sim m T\left(r, e^{L}\right)
$$

with $m=1,2,4$. Then $L$ should be a polynomial, whose degree coincides with the one of $H$. In this case we can return back $y$ from $f$. Then we have

$$
\Delta=D \cdot K^{2} \text {. }
$$

The number of poles of $K$ is finite again. This gives that the zeros of $G$ is finite in number. Hence

$$
D=\Delta \cdot \beta^{2} \cdot e^{2 M}
$$

with a rational function $\beta$. In this case we have $\gamma_{j} \neq \gamma_{k}$ for $j \neq k$.

Case 2). $\quad N_{2}(r, 0, \Delta) \sim T\left(r, e^{H}\right)$, that is, $\delta_{1} \neq \delta_{2}=\delta_{3}$. Then

$$
N_{2}(r, 0, \Delta)=N_{2}(r, 0, D) \sim m T\left(r, e^{L}\right)
$$

with $m=1,2,4$. Then $L$ should be a polynomial. Again we can return back $y$ from $f$. Then $\Delta=D \cdot K^{2}$. Similarly we have a finite number of zeros of $G$. Hence

$$
D=\Delta \beta^{2} e^{2 M} .
$$

Then the counting function of double zeros of $\Delta$ satisfies $N_{1}(r, 0, \Delta) \sim 2 T\left(r, e^{H}\right)$ and $N_{1}(r, 0, D) \sim 2 T\left(r, e^{L}\right)$. Hence $T\left(r, e^{H}\right) \sim T\left(r, e^{L}\right)$. On the other hand $T\left(r, e^{H}\right) \sim 2 T\left(r, e^{L}\right)$, because that $N_{2}(r, 0, \Delta)=N_{2}(r, 0, D)$ and $N_{1}(r, 0, D) \sim$ 
$2 T\left(r, e^{L}\right)$. This is a contradiction.

Case 3$). \Delta$ has no simple zero. Then

$$
\begin{aligned}
& -b_{1}{ }^{2}\left(x_{0} e^{L}-\gamma_{1}\right)\left(x_{0} e^{L}-\gamma_{2}\right)\left(x_{0} e^{L}-\gamma_{3}\right)\left(x_{0} e^{L}-\gamma_{4}\right) \\
& =4\left(y_{0} e^{H}-\gamma_{1}\right)^{3} \cdot G^{2} .
\end{aligned}
$$

This is a contradiction.

\section{§6. Theorems}

We shall prove the following

THEOREM 1. Let $R_{A}$ be the Riemann surface defined in $\S 2$. Assume that its discriminant $\Delta_{R_{A}}$ satisfies

$$
\Delta_{R_{A}}=4 y_{0}{ }^{3} e^{3 H}+\zeta_{2} y_{0}{ }^{2} e^{2 H}+\zeta_{1} y_{0} e^{H}+\zeta_{0}
$$

with either $\zeta_{2} \neq 0$ or $\zeta_{1} \neq 0$, where $\zeta_{2}=12 y_{2}-y_{1}{ }^{2}, \zeta_{1}=12 y_{2}{ }^{2}-18 y_{1} y_{3}-2 y_{1}{ }^{2} y_{2}$. Then $P\left(R_{A}\right)=5$.

Theorem 2. Let $R_{B}$ be the Riemann surface defined in $\S 2$. Assume that its discriminant $\Delta_{R_{B}}$ has the form

$$
\Delta_{R_{B}}=4 y_{3} y_{0}{ }^{3} e^{3 H}+\zeta_{2} y_{0}{ }^{2} e^{2 H}+\zeta_{1} y_{0} e^{H}+\zeta_{0}
$$

with either $\zeta_{2}=12 y_{1} y_{3}-y_{2}{ }^{2} \neq 0$ or $\zeta_{1}=12 y_{1}{ }^{2} y_{3}-2 y_{1} y_{2}{ }^{2}-18 y_{2} y_{3} \neq 0$. Then $P\left(R_{B}\right)=5$.

THEOREM 3. Let $R_{C}$ be the Riemann surface defined in $\S 2$. Assume that its discriminant $\Delta_{R_{C}}$ has the form

$$
\Delta_{R_{C}}=\xi_{3} y_{0}{ }^{3} e^{3 H}+\xi_{2} y_{0}{ }^{2} e^{2 H}+\xi_{1} y_{0} e^{H}+\xi_{0}
$$

with either $\xi_{2}=8 a_{2}^{2} y_{1}{ }^{2}-36 a_{2}^{3} y_{1}+27 a_{2}{ }^{4}-8 a_{2} y_{1} y_{2}+30 a_{2}{ }^{2} y_{2}-y_{2}{ }^{2} \neq 0$ or $\xi_{1}=4 a_{2}^{2} y_{1}^{3}-$ $4 a_{2} y_{1}{ }^{2} y_{2}-18 a_{2}{ }^{2} y_{1} y_{2}-2 y_{1} y_{2}{ }^{2}+24 a_{2} y_{2}{ }^{2} \neq 0$. Then $P\left(R_{C}\right)=5$.

THEOREM 4. Let $R_{D}$ be the Riemann surface defined in $\S 2$. Assume that its discriminant $\Delta_{R_{D}}$ has the form

$$
\Delta_{R_{D}}=4 y_{0}{ }^{3} e^{3 H}+\xi_{2} y_{0}{ }^{2} e^{2 H}+\xi_{1} y_{0} e^{H}+\xi_{0}
$$

with either $\xi_{2}=12 y_{2}+27 a_{2}^{2}-18 a_{2} y_{1}-y_{1}^{2} \pm 0$ or $\xi_{1}=12 y_{2}{ }^{2}-6 y_{1}^{2} y_{2}-18 a_{2} y_{1} y_{2}+4 a_{2} y_{1}^{3}$ \pm 0 . Then $P\left(R_{D}\right)=5$.

Proof of Theorem 1. Suppose that $P\left(R_{A}\right)=6$. Then on $R_{A}$ there is an entire algebroid function $f$ for which $P(f)=6$. Suppose that $f$ defines the surface $X_{1}$. Then by (4)

$$
D=\Delta_{R_{A}} \cdot \beta^{2} e^{2 M}
$$


which is just the following identity:

$$
\begin{aligned}
& -b_{1}{ }^{2} x_{0}{ }^{4} e^{4 L}+\eta_{3} x_{0}{ }^{3} e^{3 L}+\eta_{2} x_{0}{ }^{2} e^{2 L}+\eta_{1} x_{0} e^{L}+\eta_{0} \\
& =\left(4 y_{0}{ }^{3} e^{3 H}+\zeta_{2} y_{0}{ }^{2} e^{2 H}+\zeta_{1} y_{0} e^{H}+\zeta_{0}\right) \beta^{2} e^{2 H} .
\end{aligned}
$$

Now we shall make use of the unicity theorem of Borel, which plays the decisive role in our proof. Evidently we have

$$
4 T\left(r, e^{L}\right) \sim N_{2}(r, 0, D)=N_{2}\left(r, 0, \Delta_{R_{A}}\right) \sim 3 T\left(r, e^{H}\right) .
$$

We already proved that it is enough to consider this case. Hence

$$
T\left(r, e^{H}\right) \sim \frac{4}{3} T\left(r, e^{L}\right) .
$$

This relation makes our discussion simpler. Firstly assume that $M \equiv 0$. Then

$$
\begin{aligned}
& -b_{1}{ }^{2} x_{0}{ }^{4} e^{4 L}+\eta_{3} x_{0}{ }^{3} e^{3 L}+\eta_{2} x_{0}{ }^{2} e^{2 L}+\eta_{1} x_{0} e^{L}+\eta_{0} \\
& =4 \beta^{2} y_{0}{ }^{3} e^{3 H}+\beta^{2} \zeta_{2} y_{0}{ }^{2} e^{2 H}+\beta^{2} \zeta_{1} y_{0} e^{H}+\beta^{2} \zeta_{0} .
\end{aligned}
$$

There remains only one possibility: $\eta_{0}=\beta^{2} \zeta_{0},-b_{1}{ }^{2} x_{0}{ }^{4}=4 \beta^{2} y_{0}{ }^{3}, 4 L \equiv 3 H$ and $\eta_{3}=\eta_{2}=\eta_{1}=\zeta_{2}=\zeta_{1}=0$. However at least one of $\zeta_{1}$, $\zeta_{2}$ does not vanish by our assumption. Thus we arrive at a contradiction.

Next assume that $M \neq 0$. Then

$$
\begin{aligned}
& -b_{1}{ }^{2} x_{0}{ }^{4} e^{4 L}+\eta_{3} x_{0}{ }^{3} e^{3 L}+\eta_{2} x_{0}{ }^{2} e^{2 L}+\eta_{1} x_{0} e^{L}+\eta_{0} \\
& =4 \beta^{2} y_{0}{ }^{3} e^{3 H+2 M}+\beta^{2} \zeta_{2} y_{0}{ }^{2} e^{2 H+2 M}+\beta^{2} \zeta_{1} y_{0} e^{H+2 M}+\beta^{2} \zeta_{0} e^{2 M} .
\end{aligned}
$$

Now suppose that $3 H+2 M=0$. Then

$$
\begin{aligned}
& -b_{1}{ }^{2} x_{0}{ }^{4} e^{4 L}+\eta_{3} x_{0}{ }^{3} e^{3 L}+\eta_{2} x_{0}{ }^{2} e^{2 L}+\eta_{1} x_{0} e^{L}+\eta_{0} \\
& =4 \beta^{2} y_{0}{ }^{3}+\beta^{2} \zeta_{2} y_{0}{ }^{2} e^{-H}+\beta^{2} \zeta_{1} y_{0} e^{-2 H}+\beta^{2} \zeta_{0} e^{-3 H} .
\end{aligned}
$$

There remains only one possible case:

$$
\eta_{0}=4 \beta^{2} y_{0}{ }^{3}, \quad-b_{1}{ }^{2} x_{0}{ }^{4}=\beta^{2} \zeta_{0}, \quad 4 L=-3 H, \quad \eta_{3}=\eta_{2}=\eta_{1}=\zeta_{2}=\zeta_{1}=0 .
$$

This is again a contradiction. Still there are several subcases to be discussed. However all of them lead to contradictions easily.

Suppose that $f$ defines the surface $X_{2}$. Then we have

$$
D=\Delta_{R_{A}} \cdot \beta^{2} e^{2 M}
$$

by (4), which is just the following identity :

$$
\begin{aligned}
& -\left(b_{1}-b_{2}\right)^{2} x_{0}{ }^{4} e^{4 L}+\eta_{3} x_{0}{ }^{3} e^{3 L}+\eta_{2} x_{0}{ }^{2} e^{2 L}+\eta_{1} x_{0} e^{L}+\eta_{0} \\
& =\left(4 y_{0}{ }^{3} e^{3 H}+\zeta_{2} y_{0}{ }^{2} e^{2 H}+\zeta_{1} y_{0} e^{H}+\zeta_{0}\right) \beta^{2} e^{2 M} .
\end{aligned}
$$


There appear only two possible cases: Either $\eta_{0}=\beta^{2} \zeta_{0},-\left(b_{1}-b_{2}\right)^{2} x_{0}{ }^{4}=4 \beta^{2} y_{0}{ }^{3}$, $M \equiv 0,4 L \equiv 3 H$ and $\eta_{3}=\eta_{2}=\eta_{1}=\zeta_{2}=\zeta_{1}=0$ or $\eta_{0}=4 \beta^{2} y_{0}{ }^{3}-\left(b_{1}-b_{2}\right)^{2} x_{0}{ }^{4}=\beta^{2} \zeta_{0}, 2 M \equiv$ $-3 H, 4 L \equiv-3 H$ and $\eta_{3}=\eta_{2}=\eta_{1}=\zeta_{2}=\zeta_{1}=0$. These two cases give the same contradiction $\zeta_{2}=\zeta_{1}=0$. Therefore $P\left(R_{A}\right)=5$.

Proofs of Theorems 2, 3 and 4 are quite similar as in the one of Theorem 1. So we shall omit their proofs.

Theorem 5. Let $R_{E}$ be the Riemann surface defined in $\S 2$. Assume that its discriminant $\Delta_{R_{E}}$ has the form

$$
\Delta_{R_{E}}=y_{0} e^{H}\left(-a_{1}{ }^{2} y_{0}{ }^{3} e^{3 H}+A_{2} y_{0}{ }^{2} e^{2 H}+A_{1} y_{0} e^{H}+A_{0}\right),
$$

where either $A_{2}=4 a_{1}{ }^{3}-2\left(2 a_{2}+a_{3}\right) a_{1}{ }^{2}-2\left(a_{2}+2 a_{3}\right) a_{2} a_{1}+4 a_{2}{ }^{2} a_{3} \neq 0$ or $A_{1}=\left(8 a_{2}{ }^{2}+\right.$ $\left.20 a_{2} a_{3}-a_{3}{ }^{2}\right) a_{1}{ }^{2}-\left(8 a_{2}{ }^{3}+38 a_{2}{ }^{2} a_{3}+8 a_{2} a_{3}{ }^{2}\right) a_{1}-a_{2}{ }^{4}+20 a_{2}{ }^{3} a_{3}+8 a_{2}{ }^{2} a_{3} \neq 0$. Then $P\left(R_{E}\right)$ $=5$.

THEOREM 6. Let $R_{F}$ be the Riemann surface defined in $\S 2$. Assume that its discriminant $\Delta_{R_{F}}$ has the form

$$
\Delta_{R_{F}}=y_{0} e^{H}\left(-a_{1}^{2} y_{0}^{3} e^{3 H}+A_{2} y_{0}^{2} e^{2 H}+A_{1} y_{0} e^{H}+A_{0}\right)
$$

with either $A_{2}=4 a_{1}{ }^{3}-2\left(a_{2}+2 a_{3}\right) a_{1}{ }^{2}-2\left(2 a_{2}+a_{3}\right) a_{3} a_{1}+4 a_{2} a_{3}{ }^{2} \neq 0$ or $A_{1}=\left(8 a_{3}{ }^{2}+\right.$ $\left.20 a_{2} a_{3}-a_{2}{ }^{2}\right) a_{1}{ }^{2}-\left(8 a_{3}{ }^{3}+38 a_{3}{ }^{2} a_{2}+8 a_{3} a_{2}{ }^{2}\right) a_{1}-a_{3}{ }^{4}+20 a_{3}{ }^{3} a_{2}+8 a_{2}{ }^{2} a_{3}{ }^{2} \neq 0$. Then $P\left(R_{F}\right)$ $=5$.

THEOREM 7. Let $R_{G}$ be the Riemann surface defined in $\S 2$. Assume that its discriminant $\Delta_{R_{G}}$ has the form

with either

$$
\Delta_{R_{G}}=y_{0} e^{H}\left(-\left(a_{1}-a_{2}\right)^{2} y_{0}{ }^{3} e^{3 H}+A_{2} y_{0}{ }^{2} e^{2 H}+A_{1} y_{0} e^{H}+A_{0}\right)
$$

or

$$
A_{2}=-2\left(a_{1}^{2}-4 a_{1} a_{2}+a_{2}^{2}\right) a_{3}-2\left(a_{1}+a_{2}\right)\left(2 a_{1}{ }^{2}-5 a_{1} a_{2}+2 a_{2}^{2}\right) \neq 0
$$

$$
A_{1}=-\left(a_{1}{ }^{2}-10 a_{1} a_{2}+a_{2}{ }^{2}\right) a_{3}{ }^{2}-18 a_{1} a_{2}\left(a_{1}+a_{2}\right) a_{3}+27{a_{1}}^{2} a_{2}{ }^{2} \neq 0 .
$$

Then $P\left(R_{G}\right)=5$.

THEOREM 8. Let $R_{H}$ be the Riemann surface defined in $\S 2$. Assume that its discriminant $\Delta_{R_{H}}$ has the form

$$
\Delta_{R_{H}}=y_{0} e^{H}\left(-\left(a_{1}-a_{2}\right)^{2} y_{0}^{3} e^{3 H}+A_{2} y_{0}^{2} e^{2 H}+A_{1} y_{0} e^{H}+A_{0}\right)
$$

with either

$$
\begin{aligned}
A_{2}= & -2 a_{3}{ }^{2}\left(a_{1}+a_{2}\right)-4 a_{3}\left(a_{1}{ }^{2}+4 a_{1} a_{2}+a_{2}{ }^{2}\right) \\
& +2\left(a_{1}+a_{2}\right)\left(2 a_{1}{ }^{2}-5 a_{1} a_{2}+2 a_{2}{ }^{2}\right) \\
& \neq 0
\end{aligned}
$$


or

$$
\begin{aligned}
A_{1}= & -a_{3}{ }^{4}-8 a_{3}{ }^{3}\left(a_{1}+a_{2}\right)+a_{3}{ }^{2}\left(8 a_{1}{ }^{2}+46 a_{1} a_{2}+8 a_{2}{ }^{2}\right) \\
& -36 a_{1} a_{2}\left(a_{1}+a_{2}\right) a_{3}+27{a_{1}}^{2} a_{2}{ }^{2} \\
& \neq 0 .
\end{aligned}
$$

Then $P\left(R_{H}\right)=5$.

Proof of Theorem 5. Suppose that $P\left(R_{E}\right)=6$. Then on $R_{E}$ there is an entire algebroid function $f$ for which $P(f)=6$. Suppose that $f$ defines the surface $X_{1}$. Then we have

$$
D=\Delta_{R_{E}} \cdot \beta^{2} e^{2 M}
$$

by (4). This is just the following identity :

$$
\begin{aligned}
& -b_{1}{ }^{2} x_{0}{ }^{4} e^{4 L}+\eta_{3} x_{0}{ }^{3} e^{3 L}+\eta_{2} x_{0}{ }^{2} e^{2 L}+\eta_{1} x_{0} e^{L}+\eta_{0} \\
& =y_{0} e^{H}\left(-a_{1}{ }^{2} y_{0}{ }^{3} e^{3 H}+A_{2} y_{0}{ }^{2} e^{2 H}+A_{1} y_{0} e^{H}+A_{0}\right) \beta^{2} e^{2 M} .
\end{aligned}
$$

There remain only two possible cases: Either $2 M \equiv-H, 3 H \equiv 4 L, \eta_{3}=\eta_{2}=\eta_{1}=$ $A_{2}=A_{1}=0$ or $2 M \equiv-4 H, 4 L \equiv-3 H, \eta_{3}=\eta_{2}=\eta_{1}=A_{2}=A_{1}=0$. These contradict our assuption: Either $A_{2} \neq 0$ or $A_{1} \neq 0$.

Similarly we have a contradiction, when $f$ defines the surface $X_{2}$.

Proofs of Theorems 6, 7 and 8 are quite similar as in the one of Theorem 5 .

\section{§7. Unsolved problems and Remarks}

i) Let $R_{A}$ be the Riemann surface defined in $\S 2$. Assume that its discriminant $\Delta_{R_{A}}$ has the following form:

$$
\Delta_{R_{A}}=4 y_{0}^{3} e^{3 H}+\zeta_{0} .
$$

Is $P\left(R_{A}\right)$ still five?

Of course there are corresponding unsolved problems for $R_{X}(x=B, C, D$, $E, F, G, H)$.

ii) Let $R_{X}$ and $R_{Y}$ be the surfaces $P\left(R_{X}\right)=5$ and $P\left(R_{Y}\right)=5$. Can we list up all the analytic mappings of $R_{X}$ into $R_{Y}$ ?

iii) Let $R$ and $S$ be the surfaces of $P(R)=6$ and $P(S)=5$. Is there any analytic mapping of $R$ into $S$ ?

We shall now give some remarks. Let

$$
F(z, y) \equiv y^{3}-S_{1} y^{2}+S_{2} y-S_{3}=0
$$

and

$$
\alpha^{3} G(z, Y) \equiv F(z, \alpha Y+\beta)
$$




$$
=\alpha^{3}\left[Y^{3}-T_{1} Y^{2}+T_{2} Y-T_{3}\right]=0
$$

with $A_{2} \alpha=-a_{4}, A_{3} \alpha=a_{2}-a_{4}, A_{4} \alpha=a_{3}-a_{4}$ and $\beta=a_{4}$.

$R_{A}$ is defined by $F(z, y)=0$ with

$$
\left\{\begin{array}{l}
S_{1}=a_{2}+a_{3}+a_{4}, \\
S_{2}=y_{0} e^{H}+a_{2} a_{3}+a_{3} a_{4}+a_{2} a_{4}, \\
S_{3}=a_{2} a_{3} a_{4} .
\end{array}\right.
$$

Then

$$
\left\{\begin{array}{l}
T_{1}=A_{3}+A_{4}, \\
T_{2}=Y_{0} e^{H}+A_{3} A_{4}, \\
T_{3}=A_{2} Y_{0} e^{H}
\end{array}\right.
$$

with $Y_{0}=y_{0} / \alpha^{2}$. Then $G(z, Y)=0$ defines the surface $R_{D}$. Evidently inverse process is possible. Hence $R_{A}$ coincides with $R_{D}$.

Similarly we can show that $R_{B}$ coincides with $R_{C}$.

Next we put

$$
A_{1} \alpha=-a_{3}, \quad A_{2} \alpha=a_{1}-a_{3}, \quad A_{3} \alpha=a_{2}-a_{3}, \quad \beta=a_{3}
$$

$R_{E}$ is defined by $F(z, y)=0$ with

$$
\left\{\begin{array}{l}
S_{1}=2 a_{2}+a_{3}+y_{0} e^{H}, \\
S_{2}=a_{2}{ }^{2}+2 a_{2} a_{3}+a_{1} y_{0} e^{H}, \\
S_{3}=a_{2}{ }^{2} a_{3} .
\end{array}\right.
$$

Then

$$
\left\{\begin{array}{l}
T_{1}=Y_{0} e^{H}+2 A_{3}, \\
T_{2}=\left(A_{1}+A_{2}\right) Y_{0} e^{H}+A_{3}{ }^{2}, \\
T_{3}=A_{1} A_{2} Y_{0} e^{H}
\end{array}\right.
$$

with $Y_{0}=y_{0} / \alpha . \quad G(z, Y)=0$ defines the surface $R_{H}$. Hence $R_{E}$ and $R_{H}$ are coincident with each other.

Similarly we can show that $R_{F}$ and $R_{G}$ are coincident with each other. Next we put $A_{1} \alpha=-a_{1}, A_{2} \alpha=a_{3}-a_{1}, A_{3} \alpha=a_{2}-\alpha_{1}$ and $\beta=a_{1} . \quad R_{E}$ is defined by $F(z, y)=0$ with

$$
\left\{\begin{array}{l}
S_{1}=2 a_{2}+a_{3}+y_{0} e^{H}, \\
S_{2}=a_{2}{ }^{2}+2 a_{2} a_{3}+a_{1} y_{0} e^{H}, \\
S_{3}=a_{2}{ }^{2} a_{3} .
\end{array}\right.
$$

Then 


$$
\left\{\begin{array}{l}
T_{1}=Y_{0} e^{H}+A_{2}+2 A_{3}, \\
T_{2}=A_{1} Y_{0} e^{H}+2 A_{2} A_{3}+A_{3}{ }^{2}, \\
T_{3}=A_{2} A_{3}{ }^{2}
\end{array}\right.
$$

with $Y_{0}=y_{0} / \alpha$. Hence $G(z, Y)=0$ defines the surface $R_{F}$. This shows that $R_{E}$ coincides with $R_{F}$.

Therefore there are three types of Riemann surfaces of five Picard constant.

Acknowledgement. The authors wish to thank the referee for many valuable comments and suggestions.

\section{REFERENCES}

[1] G. Hiromi AND K. NinNo, On a characterization of regularly branched threesheeted covering Riemann surfaces, Kōdai Math. Sem. Rep., 17 (1965), 250-260.

[2] K. Ninno And M. Ozawa, Deficiencies of an entire algebroid function, Kōdai Math. Sem. Rep., 22 (1970), 98-113.

[3] M. Ozawa, On complex analytic mappings, Kōdai Math. Sem. Rep., 17 (1965), 93-102.

[4] M. Ozawa, On ultrahyperelliptic surfaces, Kōdai Math. Sem. Rep., 17 (1965), 103-108.

[5] M. OzAwA, Analytic mappings between three-sheeted algebroid surfaces of maximal Picard constant, in preparation.

[6] G. Rémoundos, Extension aux fonctions algébroides multiformes du théorème de M. Picard et de ses généralisations, Mém. Sci. Math. Paris 1927.

[7] H.L. Selberg, Algebroide Funktionen und Umkehrfunktionen Abelscher Integrale, Avh. Norske Vid. Akad. Oslo, 8 (1934), 1-72.

Department of Mathematics

SCIENCE UNIVERSiTy OF TOKYO

Noda, Chiba, Japan 